TRANSACTIONS OF THE

AMERICAN MATHEMATICAL SOCIETY

Volume 357, Number 8, Pages 3311-3324

S 0002-9947(05)03735-9

Article electronically published on February 4, 2005

\title{
THURSTON'S WEAK METRIC ON THE TEICHMÜLLER SPACE OF THE TORUS
}

\author{
ABDELHADI BELKHIRAT, ATHANASE PAPADOPOULOS, AND MARC TROYANOV
}

\begin{abstract}
We define and study a natural weak metric on the Teichmüller space of the torus. A similar metric has been defined by W. Thurston on the Teichmüller space of higher genus surfaces and our definition is motivated by Thurston's definition. However, we shall see that in the case of the torus, this metric has a different behaviour than on higher genus surfaces.
\end{abstract}

\section{INTRODUCTION}

For any integer $\gamma \geq 1$, let $S_{\gamma}$ be the closed oriented topological surface of genus $\gamma$. The Teichmüller space $\mathbb{T}\left(S_{\gamma}\right)$ of $S_{\gamma}$ is a space of equivalence classes of Riemannian metrics of constant curvature equal to 0 (a flat metric) in the case where $\gamma=1$ and equal to -1 (a hyperbolic metric) in the case where $\gamma \geq 2$. (We shall recall below the precise definition of the equivalence relation.) A number of important metrics on these Teichmüller spaces have been studied. One of the most interesting ones was defined by Teichmüller in the early developments of the theory. It is called the Teichmüller metric, and in this paper we shall denote it by $\tau$. The Teichmüller metric is a Finsler metric on $\mathbb{T}\left(S_{\gamma}\right)$, and it is not very difficult to see that the Teichmüller space of the torus, $\mathbb{T}\left(S_{1}\right)$, equipped with the Teichmüller metric, is isometric to the hyperbolic plane.

In the present paper, we study a weak metric on $\mathbb{T}\left(S_{1}\right)$ whose definition is inspired from a definition made by $\mathrm{W}$. Thurston in the case where the genus is $\geq 2$. Before stating our results, we recall a few classical definitions.

In this paper, we shall deal separately with the cases $\gamma \geq 2$ and $\gamma=1$ and, to simplify notation, we shall denote by $S$ a fixed closed oriented surface of genus $\gamma \geq 2$ and by $T^{2}$ the closed oriented surface of genus 1 , that is, the two-dimensional torus.

As a general motivation, we begin with the case of surfaces of genus $\geq 2$. Later on, we shall only deal with the case of genus 1 .

We recall that the Teichmüller space $\mathbb{T}(S)$ of $S$ is the set $\mathcal{H}(S)$ of hyperbolic metrics on $S$ modulo the action of the group $\operatorname{Diff}_{0}(S)$ of diffeomorphisms of $S$ isotopic to the identity by taking the pull-back of the metric. Thus, we can write

$$
\mathbb{T}(S):=\mathcal{H}(S) / \operatorname{Diff}_{0}(S) .
$$

Received by the editors February 3, 2004.

2000 Mathematics Subject Classification. Primary 30F60, 32G15.

Key words and phrases. Teichmüller space, Teichmüller metric, Thurston metric, nonsymmetric metric, weak metric, hyperbolic plane, quasi-isometry. 
The Teichmüller distance $\tau$ on $\mathbb{T}(S)$ is defined as follows:

$$
\tau\left(g_{1}, g_{2}\right)=\frac{1}{2} \inf \left\{\log K(f) \mid f \in \operatorname{Diff}_{0}(S)\right\},
$$

where $g_{1}$ and $g_{2}$ are hyperbolic metrics on $S$ representing two elements of the Teichmüller space $\mathbb{T}(S)$ and $K(f)$ the dilatation of the diffeomorphism $f$ :

$$
K(f)=\sup _{x \in S}\left(\frac{\sup \left\{\left\|d f_{x}(u)\right\| \mid u \in T_{x} S,\|u\|=1\right\}}{\inf \left\{\left\|d f_{x}(u)\right\| \mid u \in T_{x} S,\|u\|=1\right\}}\right) .
$$

In this formula, $d f_{x}$ is the differential of the diffeomorphism $f:\left(S, g_{1}\right) \rightarrow\left(S, g_{2}\right)$ at the point $x$. Of course, the norm of the tangent vector $d f_{x}(u)$ is measured with respect to the metric $g_{2}$ and the norm of the tangent vector $u$ is measured with respect to the metric $g_{1}$. Teichmüller proved in [5] that the map $\tau$ is a metric and that the Teichmüller space $\mathbb{T}(S)$, equipped with the topology induced from the metric $\tau$, is homeomorphic to $\mathbb{R}^{6 \gamma-6}$.

In 6], W. Thurston introduced and studied two alternative distances on the Teichmüller space of $S$. The first distance is defined as

$$
\lambda\left(g_{1}, g_{2}\right)=\inf \log \left\{\operatorname{Lip}(f) \mid f \in \operatorname{Diff}_{0}(S)\right\},
$$

where

$$
\operatorname{Lip}(f):=\sup \left\{\frac{d_{g_{2}}(f(x), f(y))}{d_{g_{1}}(x, y)} \mid x, y \in S, x \neq y\right\}
$$

is the Lipschitz constant of the map $f:\left(S, g_{1}\right) \rightarrow\left(S, g_{2}\right)$. This distance is a weak metric, i.e. it satisfies $\lambda(g, g)=0$ for all $g$ in $\mathbb{T}(S)$ and $0 \leq \lambda\left(g_{1}, g_{3}\right) \leqslant$ $\lambda\left(g_{1}, g_{2}\right)+\lambda\left(g_{2}, g_{3}\right)$ for all $g_{1}, g_{2}$ and $g_{3}$ in $\mathbb{T}(S)$. Furthermore, this weak metric separates points in $\mathbb{T}(S)$, that is, it satisfies $\lambda\left(g_{1}, g_{2}\right)>0$ if $g_{1} \neq g_{2}$. However, it is not a metric in the usual sense since it is not symmetric.

The second distance on $\mathbb{T}(S)$ defined by Thurston is based on the comparison of the lengths of closed curves with respect to the hyperbolic metrics $g_{1}$ and $g_{2}$. More precisely, for every isotopy class $\alpha$ of closed curves, we denote by $l_{g}(\alpha)$ the infimum of the set of lengths of closed curves $a:[0,1] \rightarrow S$ in the isotopy class $\alpha$ computed in the metric $g$ :

$$
l_{g}(\alpha)=\inf \left\{\int_{0}^{1} \sqrt{g(\dot{a}, \dot{a})} d t \mid a \in \alpha\right\} .
$$

Thurston defined the weak metric $\kappa$ on $\mathbb{T}(S)$ by setting

$$
\kappa\left(g_{1}, g_{2}\right)=\sup _{\alpha \in \mathcal{S}(S)} \log \left(\frac{l_{g_{2}}(\alpha)}{l_{g_{1}}(\alpha)}\right),
$$

where $\mathcal{S}(S)$ is the set of nontrivial isotopy classes of closed curves in the surface $S$.

One of the main results proved by Thurston in [6] is the following

Theorem 1. For every closed surface $S$ of genus $\gamma \geq 2$, the weak metrics $\kappa$ and $\lambda$ coincide on $\mathbb{T}(S)$. Furthermore, these weak metrics are nonsymmetric and they separate points:

$$
\left(\lambda\left(g_{1}, g_{2}\right)=\kappa\left(g_{1}, g_{2}\right)=0\right) \Longleftrightarrow g_{1}=g_{2} .
$$

Our goal in this paper is to study similar weak metrics $\lambda$ and $\kappa$ on the Teichmüller space of the torus $T^{2}$. Thus, we now focus our attention on the case of genus 1 .

The Teichmüller space of the torus is defined as

$$
\mathbb{T}\left(T^{2}\right)=\mathcal{F}\left(T^{2}\right) /\left(\mathbb{R}_{+}^{*} \times \operatorname{Diff}_{0}\left(T^{2}\right)\right),
$$


where $\mathcal{F}(S)$ is the set of flat metrics on $T^{2}$ and where the group $\mathbb{R}_{+}^{*}$ acts by scaling the metric, that is, multiplying it by a constant factor.

Because a flat metric remains flat after scaling, a slight modification in the definitions of $\lambda$ and $\kappa$ will be necessary to obtain well-defined weak metrics on $\mathbb{T}\left(T^{2}\right)$. With the appropriate definitions, we shall prove the following results:

- The weak metrics $\lambda$ and $\kappa$ coincide on $\mathbb{T}\left(T^{2}\right)$.

- These weak metrics are nonsymmetric.

- They do not separate points.

- These weak metrics are not quasi-isometric to the Teichmüller metric $\tau$.

- Their symmetrization is equal to the Teichmüller metric, i.e.

$$
\lambda\left(g_{1}, g_{2}\right)+\lambda\left(g_{2}, g_{1}\right)=2 \tau\left(g_{1}, g_{2}\right) .
$$

- The extremal map for $\kappa$ (the minimal stretch map in the terminology of [6]) coincides with the Teichmüller extremal map and it is the unique affine map between the two flat metrics on the torus.

In the course of proving these results, we shall give an explicit formula for the weak metric $\kappa$.

The rest of the paper is organized as follows. In Section 2, we discuss the notion of weak metric and we study in detail an example of a weak metric $\delta$ on the upper half-plane $\mathbb{H}^{2}$. This example will be a model for the weak metric $\kappa$ on $\mathbb{T}\left(T^{2}\right)$. In Section 3, we recall some basic facts about $\mathbb{T}\left(T^{2}\right)$, the Teichmüller space of the torus $T^{2}$. In Section 4, we study the weak metric $\kappa$ on $\mathbb{T}\left(T^{2}\right)$ and we show that it coincides with the metric $\delta$ defined in Section 2. Then, we show that the weak metric $\kappa$ does not separate points and is not symmetric, that it is not quasi-isometric to the Teichmüller metric and that the symmetrization of this metric coincides with

the Teichmüller metric. In Section 5, we consider the weak metric $\lambda$ on $\mathbb{T}\left(T^{2}\right)$ and we show that it coincides with the weak metric $\kappa$.

\section{THE NOTION OF WEAK METRIC}

The notion of metric space was introduced by M. Fréchet in 1906 (see 2], where a metric is called an "écart") and a number of variants of the set of axioms for a metric space have been proposed in the following decades. Nonsymmetric metrics have been studied extensively by H. Busemann; see for instance [1]. As Busemann notes, the metrics that arise in the calculus of variations, that is, the metrics that are defined by an extremal property, are in general nonsymmetric. The term weak metric was introduced by H. Ribeiro in 1943 (see 4) in relation with the metrization problem of topological spaces and Fréchet $(\mathrm{V})$ spaces, and we shall adopt this terminology.

Definitions. a) A weak metric on a set $X$ is a map $\eta: X \times X \rightarrow \mathbb{R}$ such that for all $x, y$ and $z$ in $X$, we have

1) $\eta(x, x)=0$

2) $\eta(x, y) \geq 0$

3) $\eta(x, y)+\eta(y, z) \geq \eta(x, z)$.

A weak metric $\eta$ becomes a metric if it separates points, i.e. if

$$
\eta(x, y)=0 \Longleftrightarrow x=y,
$$

and if it is symmetric, i.e. for every $x$ and $y$ in $X$

$$
\eta(x, y)=\eta(y, x) .
$$


b) We define the symmetrization of a weak metric $\eta: X \times X \rightarrow \mathbb{R}$ by the formula

$$
S \eta(x, y):=\frac{1}{2}(\eta(x, y)+\eta(y, x))
$$

for every $x$ and $y$ in $X$. This is again a weak metric and it is a metric if $\eta$ separates points.

We note that there exist other (not less) natural symmetrizations of a weak metric $\eta$, namely the weak metrics $\sigma \eta: X \times X \rightarrow \mathbb{R}$ and $\sigma^{*} \eta: X \times X \rightarrow \mathbb{R}$ defined by

and

$$
\sigma \eta(x, y)=\max (\eta(x, y), \eta(y, x))
$$

$$
\sigma^{*} \eta(x, y)=\min (\eta(x, y), \eta(y, x)) .
$$

These two symmetrizations are used by Busemann in [1].

c) Two weak metric spaces $(X, \eta)$ and $\left(X^{\prime}, \eta^{\prime}\right)$ are quasi-isometric if there exists a map $f: X \rightarrow X^{\prime}$ and two constants $c \geq 0$ and $k \geq 1$ such that for all $x$ and $y$ in $X$, we have

$$
\frac{1}{k} \eta^{\prime}(f(x), f(y))-c \leq \eta(x, y) \leq k \eta^{\prime}(f(x), f(y))+c
$$

and for all $x^{\prime}$ in $X^{\prime}$, there exists $x \in X$ such that

$$
\eta^{\prime}\left(x^{\prime}, f(x)\right)+\eta^{\prime}\left(f(x), x^{\prime}\right) \leq c .
$$

Such a map is then called a quasi-isometry

Lemma 1. Quasi-isometry is an equivalence relation among weak metric spaces.

Proof. It is obvious that the identity map is a quasi-isometry from any weak metric space to itself. We now prove the symmetry of the relation.

Let $(X, \eta)$ and $\left(X^{\prime}, \eta^{\prime}\right)$ be weak metric spaces and assume there exists a map $f: X \rightarrow X^{\prime}$ satisfying (3). By hypothesis, one can choose for any point $x^{\prime} \in X^{\prime}$ a point $x \in X$ such that $\eta^{\prime}\left(x^{\prime}, f(x)\right)+\eta^{\prime}\left(f(x), x^{\prime}\right) \leq c$. Let us call this point $x=g\left(x^{\prime}\right)$. This defines a map $g: X^{\prime} \rightarrow X$ such that

$$
\eta^{\prime}\left(x^{\prime}, f \circ g\left(x^{\prime}\right)\right)+\eta^{\prime}\left(f \circ g\left(x^{\prime}\right), x^{\prime}\right) \leq c
$$

for all $x^{\prime}$ in $X^{\prime}$. Then we have for all $x^{\prime}$ and $y^{\prime}$ in $X^{\prime}$

$$
\begin{aligned}
\eta\left(g\left(x^{\prime}\right), g\left(y^{\prime}\right)\right) & \leq k \eta^{\prime}\left(f \circ g\left(x^{\prime}\right), f \circ g\left(y^{\prime}\right)\right)+c \\
& \leq k\left(\eta^{\prime}\left(f \circ g\left(x^{\prime}\right), x^{\prime}\right)+\eta^{\prime}\left(x^{\prime}, y^{\prime}\right)+\eta^{\prime}\left(y^{\prime}, f \circ g\left(y^{\prime}\right)\right)\right)+c \\
& \leq k\left(c+\eta^{\prime}\left(x^{\prime}, y^{\prime}\right)+c\right)+c \\
& =k \eta^{\prime}\left(x^{\prime}, y^{\prime}\right)+c(2 k+1) .
\end{aligned}
$$

On the other hand, using

$$
\eta^{\prime}\left(f \circ g\left(x^{\prime}\right), f \circ g\left(y^{\prime}\right)\right) \leq k \eta\left(g\left(x^{\prime}\right), g\left(y^{\prime}\right)\right)+c,
$$

we obtain

$$
\begin{aligned}
\eta^{\prime}\left(x^{\prime}, y^{\prime}\right) & \leq \eta^{\prime}\left(x^{\prime}, f \circ g\left(x^{\prime}\right)\right)+\eta^{\prime}\left(f \circ g\left(x^{\prime}\right), f \circ g\left(y^{\prime}\right)\right)+\eta^{\prime}\left(f \circ g\left(y^{\prime}\right), y^{\prime}\right) \\
& \leq \eta^{\prime}\left(f \circ g\left(x^{\prime}\right), f \circ g\left(y^{\prime}\right)\right)+2 c \\
& \leq k \eta\left(g\left(x^{\prime}\right), g\left(y^{\prime}\right)\right)+3 c .
\end{aligned}
$$

Hence

$$
\frac{1}{k} \eta\left(g\left(x^{\prime}\right), g\left(y^{\prime}\right)\right)-c\left(2+\frac{1}{k}\right) \leq \eta^{\prime}\left(x^{\prime}, y^{\prime}\right) \leq k \eta\left(g\left(x^{\prime}\right), g\left(y^{\prime}\right)\right)+3 c .
$$


To complete the proof that $g$ is a quasi-isometry, it suffices to show that for all $x$ in $X$, there exists a point $y$ in $X^{\prime}$ such that

$$
\eta(x, g(y))+\eta(g(y), x) \leq k c+2 c .
$$

We show that this holds with $y=f(x)$. Using the fact that for all $y$ in $X^{\prime}$ we have

$$
\eta^{\prime}(y, f \circ g(y))+\eta^{\prime}(f \circ g(y), y) \leq c,
$$

we obtain

$$
\begin{aligned}
\eta(x, g(y))+\eta(g(y), x) & =\eta(x, g \circ f(x))+\eta(g \circ f(x), x) \\
& \leq k\left(\eta^{\prime}(f(x), f \circ g \circ f(x))+\eta^{\prime}(f \circ g \circ f(x), f(x))\right)+2 c \\
& \leq k c+2 c .
\end{aligned}
$$

It follows that $g: X^{\prime} \rightarrow X$ is a quasi-isometry.

Finally, the reader can easily check that if $f: X \rightarrow X^{\prime}$ and $h: X^{\prime} \rightarrow X^{\prime \prime}$ are quasi-isometries between weak metric spaces, then $h \circ f: X \rightarrow X^{\prime \prime}$ is also a quasi-isometry. This completes the proof of Lemma 1.

A basic example of weak metric is given on the half line $\mathbb{R}_{+}^{*}=(0, \infty)$ by

$$
\theta(s, t):=\max \{0, \log (t / s)\}=\left\{\begin{array}{cll}
\log \left(\frac{t}{s}\right) & \text { if } & s \leq t \\
0 & \text { if } & s \geq t .
\end{array}\right.
$$

This weak metric is clearly nonsymmetric and it does not separate points.

Remark (The topology induced by a nonsymmetric metric). The axioms for a nonnecessarily symmetric metric $\eta$ on a space $X$ that are used by Busemann in [1] also include a separation axiom $(\eta(x, y)=0 \Rightarrow x=y)$ and the following axiom which concerns the topology of the space $X$ : for every point $x$ in $X$ and for every sequence $\left(x_{n}\right)_{n \geq 0}$ of points in $X$, we have

$$
\lim _{n \rightarrow \infty} \eta\left(x, x_{n}\right)=0 \Longleftrightarrow \lim _{n \rightarrow \infty} \eta\left(x_{n}, x\right)=0 .
$$

Under these axioms, the two notions of convergence, $\eta\left(x, x_{n}\right) \rightarrow 0$ and $\eta\left(x_{n}, x\right) \rightarrow$ 0 coincide, and they amount to convergence in the usual sense with respect to the topology defined by any of the symmetrizations $S \eta, \sigma \eta$ and $\sigma^{*} \eta$ (which are genuine metrics). Let us also note that under these axioms of Busemann, the three metrics $S \eta, \sigma \eta$ and $\sigma^{*} \eta$ are equivalent. The collection of right open balls, $B_{r}(x, r)=\{y \in X \mid \eta(x, y)<r\}$, for $x$ varying in the set $X$ and $r$ varying in the set of positive reals, forms a sub-basis for the topology associated to each of the metrics $S \eta, \sigma \eta$ and $\sigma^{*} \eta$. The same result holds for the collection of left open balls $B_{l}(x, r)=\{y \in X \mid \eta(y, x)<r\}$.

Consider the upper half-plane $\mathbb{H}^{2}=\{\zeta \in \mathbb{C} \mid \operatorname{Im}(\zeta)>0\}$. We now study an explicit example of a weak metric on this space which will be a model for the Teichmüller space of the torus. We start by considering the function

$$
M: \mathbb{H}^{2} \times \mathbb{H}^{2} \rightarrow \mathbb{R}
$$

defined by

$$
M\left(\zeta, \zeta^{\prime}\right):=\sup _{x \in \mathbb{R}}\left|\frac{\zeta^{\prime}-x}{\zeta-x}\right|
$$


Proposition 1. The function

$$
\delta\left(\zeta, \zeta^{\prime}\right):=\log M\left(\zeta, \zeta^{\prime}\right)
$$

is a weak metric on $\mathbb{H}^{2}$. This weak metric does not separate points and is not symmetric.

Proof. It is clear that $\delta(\zeta, \zeta)=0$ for all $\zeta$ in $\mathbb{H}^{2}$. Observe also that for all $\zeta$ and $\zeta^{\prime}$ in $\mathbb{H}^{2}$, we have

$$
M\left(\zeta, \zeta^{\prime}\right) \geq \lim _{x \rightarrow \infty}\left|\frac{\zeta^{\prime}-x}{\zeta-x}\right|=1,
$$

hence $\delta\left(\zeta, \zeta^{\prime}\right) \geq 0$. The triangle inequality is easy to check. Indeed, we can write, for all $\zeta, \zeta^{\prime}$ and $\zeta^{\prime \prime}$ in $\mathbb{H}^{2}$,

$$
M\left(\zeta, \zeta^{\prime \prime}\right)=\sup _{x \in \mathbb{R}}\left|\frac{\zeta^{\prime \prime}-x}{\zeta-x}\right| \leq\left(\sup _{x \in \mathbb{R}}\left|\frac{\zeta^{\prime \prime}-x}{\zeta^{\prime}-x}\right|\right)\left(\sup _{x \in \mathbb{R}}\left|\frac{\zeta^{\prime}-x}{\zeta-x}\right|\right)=M\left(\zeta, \zeta^{\prime}\right) M\left(\zeta^{\prime}, \zeta^{\prime \prime}\right) .
$$

Therefore

$$
\delta\left(\zeta, \zeta^{\prime \prime}\right) \leq \delta\left(\zeta, \zeta^{\prime}\right)+\delta\left(\zeta^{\prime}, \zeta^{\prime \prime}\right)
$$

To see that $\delta$ does not separate points and is not symmetric, consider the points $\zeta=u+i s$ and $\zeta^{\prime}=u+i t$ (where $u, s, t \in \mathbb{R}, s, t>0$ ). Then it is easy to see that we have the following explicit formula:

$$
M^{2}(s i, t i)=\sup _{x \in \mathbb{R}}\left(\frac{t^{2}+x^{2}}{s^{2}+x^{2}}\right)=\left\{\begin{array}{cl}
\left(\frac{t}{s}\right)^{2} & \text { if } s \leq t \\
1 & \text { if } s \geq t
\end{array}\right.
$$

i.e.

$$
\delta(s i, t i)=\frac{1}{2} \log \left(M^{2}(s i, t i)\right)=\theta(s, t),
$$

where $\theta$ is the weak metric defined above.

It is clear that $\delta\left(\zeta, \zeta^{\prime}\right)=\delta\left(\zeta^{\prime}, \zeta\right)$ if and only if the points $\zeta$ and $\zeta^{\prime}$ in $\mathbb{H}^{2}$ have the same imaginary part.

The weak metric that we just defined is interesting notably because of the following property:

Proposition 2. The symmetrization of the weak metric $\delta$ coincides with the Poincaré metric on $\mathbb{H}^{2}$. In other words, for all $\zeta$ and $\zeta^{\prime}$ in $\mathbb{H}^{2}$, we have

$$
S \delta\left(\zeta, \zeta^{\prime}\right)=h\left(\zeta, \zeta^{\prime}\right)=\frac{1}{2} \log \left(\frac{\left|\zeta-\bar{\zeta}^{\prime}\right|+\left|\zeta-\zeta^{\prime}\right|}{\left|\zeta-\bar{\zeta}^{\prime}\right|-\left|\zeta-\zeta^{\prime}\right|}\right) .
$$

The proof of Proposition 2 uses Lemma 2 and Proposition 3 below. Lemma 2 gives an elementary formula in $\mathbb{H}^{2}$ which we shall use twice, and Proposition 3 gives an explicit fomula for the weak metric $\delta\left(\zeta, \zeta^{\prime}\right)$.

Lemma 2. For all $\zeta$ and $\zeta^{\prime}$ in $\mathbb{H}^{2}$, we have

$$
\left|\zeta^{\prime}-\bar{\zeta}\right|^{2}-\left|\zeta^{\prime}-\zeta\right|^{2}=|\zeta-\bar{\zeta}|\left|\zeta^{\prime}-\overline{\zeta^{\prime}}\right| .
$$

Proof. For $\zeta$ and $\zeta^{\prime}$ in $\mathbb{H}^{2}$, we have

$$
\begin{aligned}
\left|\zeta^{\prime}-\bar{\zeta}\right|^{2}-\left|\zeta^{\prime}-\zeta\right|^{2} & =\left(\zeta^{\prime}-\bar{\zeta}\right)\left(\bar{\zeta}^{\prime}-\zeta\right)-\left(\zeta^{\prime}-\zeta\right)\left(\bar{\zeta}^{\prime}-\bar{\zeta}\right) \\
& =-\zeta^{\prime} \zeta-\overline{\zeta^{\prime}} \bar{\zeta}+\bar{\zeta}^{\prime} \bar{\zeta}+\bar{\zeta}^{\prime} \zeta \\
& =-\left(\zeta^{\prime}-\bar{\zeta}^{\prime}\right)(\zeta-\bar{\zeta}) .
\end{aligned}
$$


We can write $\left(\zeta^{\prime}-\bar{\zeta}^{\prime}\right)=s i$ and $(\zeta-\bar{\zeta})=t i$ with $s, t>0$. Therefore, $\left(\zeta^{\prime}-\bar{\zeta}^{\prime}\right)(\zeta-\bar{\zeta})=$ st $i^{2}=-s t<0$ which gives

$$
-\left(\zeta^{\prime}-\bar{\zeta}^{\prime}\right)(\zeta-\bar{\zeta})=|\zeta-\bar{\zeta}|\left|\zeta^{\prime}-\bar{\zeta}^{\prime}\right| .
$$

Proposition 3. For all $\zeta$ and $\zeta^{\prime}$ in $\mathbb{H}^{2}$, we have

$$
\delta\left(\zeta, \zeta^{\prime}\right)=\log \left(\frac{\left|\zeta^{\prime}-\bar{\zeta}\right|+\left|\zeta^{\prime}-\zeta\right|}{|\zeta-\bar{\zeta}|}\right)
$$

Proof. The curve in $\mathbb{C}$ parametrized by $u(x):=\frac{\zeta^{\prime}-x}{\zeta-x}(x \in \mathbb{R})$ is a Euclidean circle. Let us find its center and radius.

We have

$$
u=\frac{\zeta^{\prime}-x}{\zeta-x} \Longleftrightarrow x=\frac{\zeta u-\zeta^{\prime}}{u-1},
$$

and the condition $x \in \mathbb{R}$ can be written as $x=\bar{x}$, or, equivalently,

$$
\frac{\zeta u-\zeta^{\prime}}{u-1}=\overline{\left(\frac{\zeta u-\zeta^{\prime}}{u-1}\right)}
$$

i.e.

$$
(\zeta-\bar{\zeta})|u|^{2}-\left(\zeta-\bar{\zeta}^{\prime}\right) u+\left(\bar{\zeta}-\zeta^{\prime}\right) \bar{u}+\left(\zeta^{\prime}-\bar{\zeta}^{\prime}\right)=0 .
$$

One can rewrite this relation as

$$
|u|^{2}-\left(\frac{\zeta-\overline{\zeta^{\prime}}}{\zeta-\bar{\zeta}}\right) u-\left(\frac{\bar{\zeta}-\zeta^{\prime}}{\bar{\zeta}-\zeta}\right) \bar{u}+\left(\frac{\zeta^{\prime}-\bar{\zeta}^{\prime}}{\zeta-\bar{\zeta}}\right)=0
$$

or, after a small calculation that uses Lemma 2 ,

$$
\left[u-\left(\frac{\bar{\zeta}-\zeta^{\prime}}{\bar{\zeta}-\zeta}\right)\right] \overline{\left[u-\left(\frac{\bar{\zeta}-\zeta^{\prime}}{\bar{\zeta}-\zeta}\right)\right]}=\left|\frac{\zeta-\zeta^{\prime}}{\zeta-\bar{\zeta}}\right|^{2} .
$$

This is the equation of a circle whose center and radius are respectively

$$
c=\left(\frac{\bar{\zeta}-\zeta^{\prime}}{\bar{\zeta}-\zeta}\right) \quad \text { and } \quad R=\left|\frac{\zeta-\zeta^{\prime}}{\zeta-\bar{\zeta}}\right| .
$$

The quantity $M\left(\zeta, \zeta^{\prime}\right)=\sup _{x \in \mathbb{R}}|u(x)|$ is the largest Euclidean distance from a point on that circle to the origin, i.e.

$$
M\left(\zeta, \zeta^{\prime}\right)=\sup _{x \in \mathbb{R}}|u(x)|=|c|+R=\frac{\left|\zeta-\bar{\zeta}^{\prime}\right|}{|\zeta-\bar{\zeta}|}+\frac{\left|\zeta-\zeta^{\prime}\right|}{|\zeta-\bar{\zeta}|},
$$

and thus

$$
\begin{aligned}
\delta\left(\zeta, \zeta^{\prime}\right) & =\log \left(\frac{\left|\zeta-\bar{\zeta}^{\prime}\right|+\left|\zeta-\zeta^{\prime}\right|}{|\zeta-\bar{\zeta}|}\right) \\
& =\log \left(\frac{\left|\zeta^{\prime}-\bar{\zeta}\right|+\left|\zeta^{\prime}-\zeta\right|}{|\zeta-\bar{\zeta}|}\right)
\end{aligned}
$$


Proof of Proposition 2. Lemma 2 implies that

$$
\begin{aligned}
& \left(\frac{\left|\zeta^{\prime}-\bar{\zeta}\right|+\left|\zeta^{\prime}-\zeta\right|}{|\zeta-\bar{\zeta}|}\right)\left(\frac{\left|\zeta-\bar{\zeta}^{\prime}\right|+\left|\zeta-\zeta^{\prime}\right|}{\left|\zeta^{\prime}-\bar{\zeta}^{\prime}\right|}\right) \\
& =\frac{\left(\left|\zeta^{\prime}-\bar{\zeta}\right|+\left|\zeta^{\prime}-\zeta\right|\right)\left(\left|\zeta-\bar{\zeta}^{\prime}\right|+\left|\zeta-\zeta^{\prime}\right|\right)}{\left|\zeta^{\prime}-\bar{\zeta}\right|^{2}-\mid \zeta^{\prime}-\zeta^{2}} \\
& =\frac{\left(\left|\zeta^{\prime}-\bar{\zeta}\right|+\left|\zeta^{\prime}-\zeta\right|\right)\left(\left|\zeta-\bar{\zeta}^{\prime}\right|+\left|\zeta-\zeta^{\prime}\right|\right)}{\left(\left|\zeta^{\prime}-\bar{\zeta}\right|-\left|\zeta^{\prime}-\zeta\right|\right)\left(\left|\zeta^{\prime}-\bar{\zeta}\right|+\left|\zeta^{\prime}-\zeta\right|\right)} \\
& =\frac{\left|\zeta-\overline{\zeta^{\prime}}\right|+\left|\zeta-\zeta^{\prime}\right|}{\left|\zeta^{\prime}-\bar{\zeta}\right|-\left|\zeta^{\prime}-\zeta\right|} .
\end{aligned}
$$

Thus, we have by Proposition 3 ,

$$
\begin{aligned}
S \delta\left(\zeta, \zeta^{\prime}\right) & =\frac{1}{2}\left(\delta\left(\zeta, \zeta^{\prime}\right)+\delta\left(\zeta^{\prime}, \zeta\right)\right) \\
& =\frac{1}{2} \log \left(\frac{\left|\zeta^{\prime}-\bar{\zeta}\right|+\left|\zeta^{\prime}-\zeta\right|}{|\zeta-\bar{\zeta}|}\right)+\frac{1}{2} \log \left(\frac{\left|\zeta-\overline{\zeta^{\prime}}\right|+\left|\zeta-\zeta^{\prime}\right|}{\left|\zeta^{\prime}-\bar{\zeta}^{\prime}\right|}\right) . \\
& =\frac{1}{2} \log \left(\frac{\left|\zeta-\bar{\zeta}^{\prime}\right|+\left|\zeta-\zeta^{\prime}\right|}{\left|\zeta-\bar{\zeta}^{\prime}\right|-\left|\zeta-\zeta^{\prime}\right|}\right) \\
& =h\left(\zeta, \zeta^{\prime}\right) .
\end{aligned}
$$

We close this section with the following observation:

Proposition 4. The two spaces $\left(\mathbb{H}^{2}, \delta\right)$ and $\left(\mathbb{H}^{2}, h\right)$ are not quasi-isometric.

The proof is based on the following, somewhat strange, lemma:

Lemma 3. For every $\zeta$ and $\xi$ in $\mathbb{H}^{2}$ and for all $\varepsilon>0$, we can find $\xi^{\prime}$ and $\zeta^{\prime}$ in $\mathbb{H}^{2}$ such that

$$
\delta\left(\zeta^{\prime}, \zeta\right)+\delta\left(\zeta^{\prime}, \xi^{\prime}\right)+\delta\left(\xi^{\prime}, \xi\right) \leq \varepsilon .
$$

Let us note that this lemma is possible because the weak metric $\delta$ is not symmetric, otherwise it would contradict the triangle inequality.

Proof. For all $s \geq 1$, let us set $\zeta_{s}=\zeta+i s$ and $\xi_{s}=\xi+i s$. Then we have by (5)

$$
\delta\left(\xi_{s}, \xi\right)=\delta\left(\zeta_{s}, \zeta\right)=\theta(1+s, 1)=0 .
$$

On the other hand, we have

$$
\lim _{s \rightarrow+\infty}\left(\frac{\left|\xi_{s}-\bar{\zeta}_{s}\right|+\left|\xi_{s}-\zeta_{s}\right|}{\left|\zeta_{s}-\bar{\zeta}_{s}\right|}\right)=\lim _{s \rightarrow+\infty}\left(\frac{|\xi-\bar{\zeta}+2 i s|+|\xi-\zeta|}{|\zeta-\bar{\zeta}+2 i s|}\right)=1,
$$

hence $\lim _{s \rightarrow+\infty} \delta\left(\zeta_{s}, \xi_{s}\right)=0$ and it suffices to take $\zeta^{\prime}=\zeta_{s}$ and $\xi^{\prime}=\xi_{s}$ with $s$ large enough.

Proof of Propositon 4. We proceed by contradiction. Suppose there exists a quasiisometry $f:\left(\mathbb{H}^{2}, h\right) \rightarrow\left(\mathbb{H}^{2}, \delta\right)$. Then there exists $k \geq 1$ and $c \geq 0$ such that

$$
\frac{1}{k} \delta(\zeta, \xi)-c \leq h(f(\zeta), f(\xi)) \leq k \delta(\zeta, \xi)+c
$$

for all $\zeta$ and $\xi$ in $\mathbb{H}^{2}$. 
Let us choose $\zeta$ and $\xi$ in $\mathbb{H}^{2}$ such that $\delta(\zeta, \xi)>1+4 k c$. By Lemma 3, we can find points $\xi^{\prime}$ and $\zeta^{\prime}$ in $\mathbb{H}^{2}$ such that $\delta\left(\zeta^{\prime}, \zeta\right)+\delta\left(\zeta^{\prime}, \xi^{\prime}\right)+\delta\left(\xi^{\prime}, \xi\right) \leq \varepsilon<1 / k^{2}$. Therefore we have

$$
\begin{aligned}
\delta(\zeta, \xi) & \leq k h(f(\zeta), f(\xi))+k c \\
& \leq k\left[h\left(f(\zeta), f\left(\zeta^{\prime}\right)\right)+h\left(f\left(\zeta^{\prime}\right), f\left(\xi^{\prime}\right)\right)+h\left(f\left(\xi^{\prime}\right), f(\xi)\right)\right]+k c \\
& =k\left[h\left(f\left(\zeta^{\prime}\right), f(\zeta)\right)+h\left(f\left(\zeta^{\prime}\right), f\left(\xi^{\prime}\right)\right)+h\left(f\left(\xi^{\prime}\right), f(\xi)\right)\right]+k c \\
& \leq k^{2}\left[\delta\left(\zeta^{\prime}, \zeta\right)+\delta\left(\zeta^{\prime}, \xi^{\prime}\right)+\delta\left(\xi^{\prime}, \xi\right)\right]+4 k c \\
& \leq k^{2} \epsilon+4 k c<1+4 k c<\delta(\zeta, \xi)
\end{aligned}
$$

which is a contradiction. Thus, such a quasi-isometry does not exist.

\section{The Teichmüller SPACE OF the torus}

We recall that we denote by $\mathcal{F}\left(T^{2}\right)$ the set of flat metrics on the 2-torus $T^{2}$. It is clear that if $g \in \mathcal{F}\left(T^{2}\right)$ and $t>0$, then $t g$ is again a flat metric. We denote by $\mathbb{P}\left(T^{2}\right)$ the quotient space $\mathcal{F}\left(T^{2}\right) / \mathbb{R}_{+}^{*}$. Equivalently, $\mathbb{P}\left(T^{2}\right)$ can be regarded as the set of flat metrics of area one on $T^{2}$. The Teichmüller space of the torus is then defined as

$$
\mathbb{T}\left(T^{2}\right):=\mathbb{P}\left(T^{2}\right) / \operatorname{Diff}_{0}\left(T^{2}\right)
$$

where $\operatorname{Diff}_{0}\left(T^{2}\right)$ is the group of diffeomorphisms of $T^{2}$ which are isotopic to the identity.

The Teichmüller space $\mathbb{T}\left(T^{2}\right)$ can be naturally identified with the hyperbolic plane $\mathbb{H}^{2}$; we shall give below two natural ways to see this identification.

In the first such identification, we associate to each complex number $\mu$ satisfying $|\mu|<1$, the flat metric on the complex plane $\mathbb{C}$ given by

$$
g_{\mu}:=|d z+\mu d \bar{z}|^{2} \text {. }
$$

This flat metric is invariant under all translations of $\mathbb{C}$ and can thus be seen as a flat metric on the torus

$$
T^{2}=\mathbb{C} /(\mathbb{Z}+i \mathbb{Z})
$$

This defines a map

$$
\begin{aligned}
\iota: \mathbb{D}^{2}=\{\mu \in \mathbb{C}|| \mu \mid<1\} & \rightarrow \mathbb{T}\left(T^{2}\right) \\
\mu & \mapsto\left[g_{\mu}\right]
\end{aligned}
$$

which is a homeomorphism. This map is an isometry between the disk $\mathbb{D}^{2}$ equipped with the Poincaré metric and the space $\mathbb{T}\left(T^{2}\right)$ equipped with the Teichmüller metric (see the remark after Theorem 2 below).

To explain the other identifications of $\mathbb{T}\left(T^{2}\right)$ with the hyperbolic plane, we need to introduce another (equivalent) definition of $\mathbb{T}\left(T^{2}\right)$ based on the notion of marked surfaces.

Definition 1. A marking of the torus is a choice of a group isomorphism $\psi: \mathbb{Z}^{2} \rightarrow$ $\pi_{1}\left(T^{2}\right)$.

Because $\pi_{1}\left(T^{2}\right)$ is abelian, there is a specific one-to-one correspondence between the group $\mathbb{Z}^{2}$ and the set of isotopy classes of closed curves in a marked torus $T^{2}$.

Definition 2. Given a marked torus, we shall denote by $\epsilon$ the isotopy class corresponding to the generator $(1,0) \in \mathbb{Z}^{2}$. 
It is a standard fact that the Teichmüller space $\mathbb{T}\left(T^{2}\right)$ can also be defined as the set of equivalence classes of triples $(S, g, \psi)$, where $S$ is a closed oriented surface of genus $1, g$ is a flat metric on $S$ and $\psi$ is a marking of $S$ and where $(S, g, \psi)$ and $\left(S^{\prime}, g^{\prime}, \psi^{\prime}\right)$ are equivalent if there exists a diffeomorphism $f: S \rightarrow S^{\prime}$ and a constant $c>0$ such that $f^{*} g^{\prime}=c g$ and $f_{*} \psi=\psi^{\prime}$.

Let us now explain our second identification of $\mathbb{T}\left(T^{2}\right)$ with the hyperbolic plane $\mathbb{H}^{2}$. Recall that the universal cover of a flat torus is the plane $\mathbb{R}^{2}$ equipped with the Euclidean metric. In this picture, a flat torus appears as the quotient of $\mathbb{R}^{2}$ by the action of a translation group

$$
\Gamma_{\zeta^{\prime}, \zeta}=\left\{m t_{\zeta^{\prime}}+n t_{\zeta} \mid m, n \in \mathbb{Z}^{2}\right\},
$$

where $\zeta^{\prime}$ and $\zeta$ are linearly independent vectors in the plane. Here, $t_{\zeta}$ (respectively $t_{\zeta^{\prime}}$ ) denotes the translation of the plane by the vector $\zeta$ (respectively $\zeta^{\prime}$ ). Performing a homothety if necessary, one may assume that $\left[\zeta^{\prime}, \zeta\right]$ is a parallelogram of area 1 .

The quotient surface $\mathbb{R}^{2} / \Gamma_{\zeta^{\prime}, \zeta}$ carries a flat metric of area 1 and a canonical marking obtained by identifying $(m, n) \in \mathbb{Z}^{2}$ with $m t_{\zeta^{\prime}}+n t_{\zeta} \in \Gamma=\pi_{1}\left(\mathbb{R}^{2} / \Gamma\right)$.

Let us now consider the two-by-two matrix whose columns are the coordinates of $\zeta^{\prime}$ and $\zeta$. Since the parallelogram $\left[\zeta^{\prime}, \zeta\right]$ spanned by these vectors has area one, the determinant of this matrix is equal to one and therefore this matrix belongs to $S L(2, \mathbb{R})$. Two such pairs of vectors give equivalent flat tori if and only if they differ by a rotation. In this way the Teichmüller space $\mathbb{T}\left(T^{2}\right)$ is naturally identified with the homogeneous space $S L(2, \mathbb{R}) / S O(2)$. It is well known that this homogeneous space has a unique left-invariant Riemannian metric and that this space, equipped with this metric, is isometric to the upper half-plane $\mathbb{H}^{2}$ equipped with the hyperbolic metric.

In the sequel, we shall identify $\mathbb{R}^{2}$ with $\mathbb{C}$ and we shall replace the normalization Area $\left[\zeta^{\prime}, \zeta\right]=1$ by the condition $\left|\zeta^{\prime}\right|=1$. We shall also fix the rotation by assuming $\zeta^{\prime}=1$ and $\operatorname{Re}(\zeta)>0$. The surface $\mathbb{R}^{2} / \Gamma_{1, \zeta}$ is then given by

$$
S_{\zeta}=\mathbb{C} /(\mathbb{Z}+\mathbb{Z} \zeta)
$$

it has a canonical flat metric and marking.

Theorem 2. The map

$$
j: \mathbb{H}^{2} \rightarrow \mathbb{T}\left(T^{2}\right)
$$

which associates to each element $\zeta \in \mathbb{H}^{2}$ the equivalence class $\left[S_{\zeta}\right]$ of the flat marked torus $S_{\zeta}$ is an isometry if $\mathbb{H}^{2}$ is equipped with the Poincaré metric and $\mathbb{T}\left(T^{2}\right)$ with the Teichmüller metric.

The proof is given in [3. Theorem 6.4, p. 219].

A calculation shows that the surface $\mathbb{C} /(\mathbb{Z}+i \mathbb{Z})$ with the flat metric $g_{\mu}=$ $|d z+\mu d \bar{z}|^{2}$ is homothetic to $S_{\zeta}=\mathbb{C} /(\mathbb{Z}+\mathbb{Z} \zeta)$ if and only if $\mu=\frac{\zeta-i}{\zeta+i}$. This implies that the following diagram commutes:

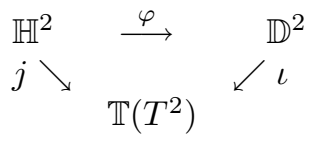

where $\varphi$ is the Cayley map $\varphi: \zeta \rightarrow \mu=\frac{\zeta-i}{\zeta+i}$. Since $\phi$ is an isometry between the upper half-plane model $\mathbb{H}^{2}$ and the unit disk model $\mathbb{D}^{2}$ of the hyperbolic plane, the map $\iota: \mathbb{D}^{2} \rightarrow \mathbb{T}\left(T^{2}\right)$ defined above is also an isometry. 
In the sequel, we shall identify the point $\zeta \in \mathbb{H}^{2}$ with the element $j(\zeta)=\left[S_{\zeta}\right] \in$ $\mathbb{T}\left(T^{2}\right)$. Recall that the surface $S_{\zeta}=\mathbb{C} /(\mathbb{Z}+\mathbb{Z} \zeta)$ has a canonical marking and observe that the image of the interval $[0,1] \subset \mathbb{C}$ via the canonical projection $\mathbb{C} \rightarrow S_{\zeta}$ is a curve of length one which represents the isotopy class $\epsilon$ (see Definition 2 above).

\section{THE WEAK METRIC $\kappa$ ON $\mathbb{T}\left(T^{2}\right)$}

Thurston's weak metric $\kappa$ which we recalled in Section 1 is not invariant under multiplication of the metrics $g_{1}$ or $g_{2}$ by positive constants. Thus, we need to modify the definition in order to obtain a similar weak metric on $\mathbb{T}\left(T^{2}\right)$.

Consider the set $\mathcal{S}\left(T^{2}\right)$ of nontrivial isotopy classes of closed curves in the surface $T^{2}$.

To any pair of metrics $g_{1}, g_{2}$ on $T^{2}$, we associate the number

$$
\kappa\left(g_{1}, g_{2}\right)=\sup _{\alpha \in \mathcal{S}\left(T^{2}\right)} \log \left(\frac{l_{g_{2}}(\alpha) / l_{g_{2}}(\epsilon)}{l_{g_{1}}(\alpha) / l_{g_{1}}(\epsilon)}\right)
$$

where $\epsilon \in \mathcal{S}\left(T^{2}\right)$ is the isotopy class defined above.

It is clear that this quantity is invariant under the action of $\mathbb{R}_{+}^{*}$ and $\operatorname{Diff}_{0}\left(T^{2}\right)$. In particular $\kappa$ defines a map

$$
\kappa: \mathbb{T}\left(T^{2}\right) \times \mathbb{T}\left(T^{2}\right) \rightarrow \mathbb{R}
$$

which is a weak metric.

Our next result states that, under the identification $j: \mathbb{H}^{2} \rightarrow \mathbb{T}\left(T^{2}\right)$, the weak metric $\kappa$ coincides with the weak metric $\delta$ on $\mathbb{H}^{2}$ defined in Section 2.

Theorem 3. For any $\zeta, \zeta^{\prime} \in \mathbb{H}^{2}$, we have

$$
\kappa\left(\zeta, \zeta^{\prime}\right)=\delta\left(\zeta, \zeta^{\prime}\right)=\log \left(M\left(\zeta, \zeta^{\prime}\right)\right)
$$

Proof. As before, we identify $\mathbb{Z}^{2}$ with $\mathcal{S}\left(T^{2}\right)$ : the isotopy class corresponding to $(m, n) \in \mathbb{Z}^{2}$ is given by the image in $S_{\zeta}=\mathbb{C} /(\mathbb{Z}+\mathbb{Z} \zeta)$ of an arbitrary path connecting $0 \in \mathbb{C}$ to $m+n \zeta$.

It is clear that the minimal length of such a path in its isotopy class is equal to $|m+n \zeta|:$

$$
\ell_{\rho_{\zeta}}(m, n)=|m+n \zeta|
$$

where we denote by $\rho_{\zeta}$ the projection of the canonical metric of $\mathbb{C}$ on the surface $S_{\zeta}=\mathbb{C} /(\mathbb{Z}+\mathbb{Z} \zeta)$.

We also have

$$
\ell_{\rho_{\zeta^{\prime}}}(m, n)=\left|m+n \zeta^{\prime}\right|
$$

and, in particular,

$$
\ell_{\rho_{\zeta}}(\epsilon)=\ell_{\rho_{\zeta^{\prime}}}(\epsilon)=\ell_{\rho}(1,0)=1
$$


Consequently

$$
\begin{aligned}
\kappa\left(\zeta, \zeta^{\prime}\right) & =\log \sup _{\alpha \in \mathcal{S}}\left(\frac{\ell_{\rho_{\zeta^{\prime}}}(\alpha) / \ell_{\rho_{\zeta^{\prime}}}(\epsilon)}{\ell_{\rho_{\zeta}}(\alpha) / \ell_{\rho_{\zeta}}(\epsilon)}\right) \\
& =\log \sup _{m, n \in \mathbb{Z}}\left(\frac{\left|m+n \zeta^{\prime}\right|}{|m+n \zeta|}\right) \\
& =\log \sup _{m, n \in \mathbb{Z}}\left(\frac{\left|(m / n)+\zeta^{\prime}\right|}{|(m / n)+\zeta|}\right) \\
& =\log \sup _{q \in \mathbb{Q}}\left(\frac{\left|q+\zeta^{\prime}\right|}{|q+\zeta|}\right) \\
& =\log \sup _{x \in \mathbb{R}}\left(\frac{\left|x+\zeta^{\prime}\right|}{|x+\zeta|}\right) \\
& =\log \left(M\left(\zeta, \zeta^{\prime}\right)\right) \\
& =\delta\left(\zeta, \zeta^{\prime}\right) .
\end{aligned}
$$

From the results of Section 2, we deduce the following

Corollary 1. (a) The weak metric $\kappa$ does not separate points and is not symmetric.

(b) The symmetrization $S \kappa$ of $\kappa$ coincides with the Teichmüller metric $\tau$.

(c) The weak metric $\kappa$ is not quasi-isometric to the Teichmüller metric $\tau$. 3

Recall also that an explicit formula for $\delta$, and hence for $\kappa$, is given in Proposition

\section{The WEAK MEtric $\lambda$ ON $\mathbb{T}\left(T^{2}\right)$}

Let us define the normalized weak Lipschitz distance between two Riemannian metrics $g_{1}, g_{2}$ on the torus $T^{2}$ by

$$
\lambda\left(g_{1}, g_{2}\right):=\inf _{\varphi}(\log \mathcal{L}(\varphi)),
$$

where $\varphi: T^{2} \rightarrow T^{2}$ is a homeomorphism isotopic to the identity and

$$
\mathcal{L}(\varphi)=\sup _{x \neq y}\left(\frac{d_{g_{2}}(\varphi(x), \varphi(y)) / l_{g_{2}}(\epsilon)}{d_{g_{1}}(x, y) / l_{g_{1}}(\epsilon)}\right) .
$$

Observe that $\lambda\left(g_{1}, g_{2}\right)$ is invariant under the action of the groups $\mathbb{R}_{+}^{*}$ and $\operatorname{Diff}{ }_{0}\left(T^{2}\right)$, that is,

$$
\lambda\left(t \phi^{*} g_{1}, s \psi^{*} g_{2}\right)=\lambda\left(g_{1}, g_{2}\right)
$$

for all $t, s \in \mathbb{R}_{+}^{*}$ and $\phi, \psi \in \operatorname{Diff}_{0}\left(T^{2}\right)$. In particular, $\lambda$ is well defined on the Teichmüller space of the torus

$$
\lambda: \mathbb{T}\left(T^{2}\right) \times \mathbb{T}\left(T^{2}\right) \rightarrow \mathbb{R},
$$

and it is easy to check that it is a weak metric.

Theorem 4. Up to identifying the spaces $\mathbb{T}\left(T^{2}\right)$ and $\mathbb{H}^{2}$ via the map $j$, we have

$$
\lambda\left(\zeta, \zeta^{\prime}\right)=\kappa\left(\zeta, \zeta^{\prime}\right)
$$

for all $\zeta$ and $\zeta^{\prime}$ in $\mathbb{T}\left(T^{2}\right)$. 
To prove the theorem, we shall use the following lemma:

Lemma 4. The Lipschitz constant of the map $\psi: \mathbb{C} \rightarrow \mathbb{C}$ defined by $\psi(\zeta):=$ $\bar{p}^{2} \zeta+q^{2} \bar{\zeta}$ (where $p, q \in \mathbb{C}$ ) is given by

$$
\operatorname{Lip}(\psi)=|p|^{2}+|q|^{2} .
$$

Proof. For all $\zeta, \zeta^{\prime} \in \mathbb{C}$, we have

$$
\left|\psi(\zeta)-\psi\left(\zeta^{\prime}\right)\right|=\left|\bar{p}^{2}\left(\zeta-\zeta^{\prime}\right)+q^{2}\left(\zeta-\zeta^{\prime}\right)\right| \leq\left(|p|^{2}+|q|^{2}\right)\left|\zeta-\zeta^{\prime}\right|,
$$

thus $\operatorname{Lip}(\psi) \leq|p|^{2}+|q|^{2}$.

To prove the converse inequality, let us choose $\zeta=p q$ and $\zeta^{\prime}=0$. We then have $\psi\left(\zeta^{\prime}\right)=0$ and

consequently

$$
\psi(\zeta)=\bar{p}^{2} p q+q^{2} \overline{p q}=\bar{p} q\left(|p|^{2}+|q|^{2}\right),
$$

$$
\left|\frac{\psi(\zeta)-\psi\left(\zeta^{\prime}\right)}{\zeta-\zeta^{\prime}}\right|=\frac{\left|\bar{p} q\left(|p|^{2}+|q|^{2}\right)\right|}{|p q|}=|p|^{2}+|q|^{2},
$$

which shows that $\operatorname{Lip}(\psi) \geq|p|^{2}+|q|^{2}$. Therefore we have $\operatorname{Lip}(\psi)=|p|^{2}+|q|^{2}$.

Proof of Theorem 4. First observe that for any homeomorphism $\varphi:\left(S, g_{1}\right) \rightarrow$ $\left(S^{\prime}, g_{2}\right)$, and any curve $c: I \rightarrow S$, we have

$$
l_{g_{2}}(\varphi \circ c) \leq \operatorname{Lip}(\varphi) \cdot l_{g_{1}}(c) .
$$

Therefore if $\varphi:\left(S_{\zeta}, \rho_{\zeta}\right) \rightarrow\left(S_{\zeta^{\prime}}, \rho_{\zeta^{\prime}}\right)$ is a marking-preserving homeomorphism, we have

$$
\kappa\left(\rho_{\zeta}, \rho_{\zeta^{\prime}}\right) \leq \lambda\left(\rho_{\zeta}, \rho_{\zeta^{\prime}}\right)
$$

(since $\ell_{\rho_{\zeta}}(\epsilon)=\ell_{\rho_{\zeta^{\prime}}}(\epsilon)=1$ and thus $\operatorname{Lip}(\varphi)=\mathcal{L}(\varphi)$ in this case).

To prove the converse inequality, we consider the map defined by

$$
\tilde{f}(x):=\left(\frac{\zeta^{\prime}-\bar{\zeta}}{\zeta-\bar{\zeta}}\right) x-\left(\frac{\zeta^{\prime}-\zeta}{\zeta-\bar{\zeta}}\right) \bar{x}
$$

This is the unique real affine map $\tilde{f}: \mathbb{C} \rightarrow \mathbb{C}$ such that

$$
\tilde{f}(0)=0, \quad \tilde{f}(1)=1 \quad \text { and } \quad \tilde{f}(\zeta)=\zeta^{\prime} .
$$

This map defines a diffeomorphism $f:\left(S_{\zeta}, \rho_{\zeta}\right) \rightarrow\left(S_{\zeta^{\prime}}, \rho_{\zeta^{\prime}}\right)$.

Applying Lemma 4 with

$$
\bar{p}^{2}=\left(\frac{\zeta^{\prime}-\bar{\zeta}}{\zeta-\bar{\zeta}}\right) \quad \text { and } \quad q^{2}=\left(\frac{\zeta^{\prime}-\zeta}{\zeta-\bar{\zeta}}\right)
$$

we see that

$$
\mathcal{L}(f)=\operatorname{Lip}(f)=\left|\frac{\zeta^{\prime}-\bar{\zeta}}{\zeta-\bar{\zeta}}\right|+\left|\frac{\zeta^{\prime}-\zeta}{\zeta-\bar{\zeta}}\right| .
$$

Using Proposition 3 and Theorem 3 , we finally have

$$
\lambda\left(\rho_{\zeta}, \rho_{\zeta^{\prime}}\right) \leq \log \left(\frac{\left|\zeta^{\prime}-\bar{\zeta}\right|+\left|\zeta^{\prime}-\zeta\right|}{|\zeta-\bar{\zeta}|}\right)=\delta\left(\zeta, \zeta^{\prime}\right)=\kappa\left(\rho_{\zeta}, \rho_{\zeta^{\prime}}\right)
$$

This completes the proof of Theorem 4 . 
Our final result is the following.

Theorem 5. The map $f:\left(S_{\zeta}, \rho_{\zeta}\right) \rightarrow\left(S_{\zeta^{\prime}}, \rho_{\zeta^{\prime}}\right)$ whose universal cover is the affine map (8) is the extremal map for the weak distance $\lambda$, i.e.

$$
\log (\mathcal{L}(f))=\lambda\left(\zeta, \zeta^{\prime}\right)
$$

It is also extremal for the Teichmüller metric, i.e.

$$
\frac{1}{2} \log (K(f))=\tau\left(\zeta, \zeta^{\prime}\right) .
$$

Proof. The first assertion follows from the proof of the previous theorem.

To prove the second assertion, recall that the dilatation of the affine map $g$ : $\mathbb{C} \rightarrow \mathbb{C}$ given by $g(x)=a x+b \bar{x}$ is given by

$$
K(g)=\frac{|a|+|b|}{|a|-|b|}
$$

(this is an easy computation, see [3, p. 19]). Applying this formula to our mapping defined by (8), we find that

$$
K(f)=\frac{\left|\zeta^{\prime}-\bar{\zeta}\right|+\left|\zeta^{\prime}-\zeta\right|}{\left|\zeta^{\prime}-\bar{\zeta}\right|-\left|\zeta^{\prime}-\zeta\right|}
$$

Hence

$$
\frac{1}{2} \log K(f)=h\left(\zeta, \zeta^{\prime}\right)=\tau\left(\zeta, \zeta^{\prime}\right)
$$

The extremal map for the Teichmüller distance is known to be unique (see [3. Theorem 6.3]).

\section{REFERENCES}

[1] Busemann, H., Local metric geometry, Trans. Amer. Math. Soc. 56 (1944) $200-274$. MR0010976 (6:97f)

[2] Fréchet, M. Sur quelques points de calcul fonctionnel, Rend. Circ. Mat. Palermo 22 (1906), $1-74$.

[3] Lehto 0., Univalent functions and Teichmüller space, Springer-Verlag (1987). MR0867407 (88f:30073)

[4] Ribeiro, H. Sur les espaces à métrique faible, Portugaliae Math. 4 (1943) 21-40. MR0009432 $(5: 149 \mathrm{~b})$

[5] Teichmüller, O. Extremale quasikonforme Abbildungen und quadratische Differentiale, Abh. Preuss. Akad. Wiss. Math.-Nat. Kl. 22 (1939). MF0003242 (2:187a)

[6] Thurston, W.P. Minimal Stretch maps between hyperbolic surfaces, Preprint (1985) available at http://arxiv.org/abs/math.GT/9801039.

Département de Recherche Mathématique Avancée, Université Louis Pasteur and CNRS, 7 rue René Descartes, 67084 Strasbourg Cedex, France

E-mail address: belkhirat@math.u-strasbg.fr

Current address: King Fahd University of Petroleum and Minerals, Hail Community College, P.O. Box 2440, Hail, Saudi Arabia

E-mail address: belkhirat@hcc.kfupm.edu.sa

Département de Recherche Mathématique Avancée, Université Louis Pasteur and CNRS, 7 rue René Descartes, 67084 Strasbourg Cedex, France

E-mail address: papadopoulos@math.u-strasbg.fr

Section de Mathématiques, École Polytechnique Féderale de Lausanne, 1015 LauSANNE, SWITZERLAND

E-mail address: marc.troyanov@epfl.ch 